\title{
The Structural Response of Gadolinium Phosphate to Pressure
}

\author{
Karina M. Heffernan, ${ }^{a}$ Nancy L. Ross ${ }^{\mathrm{a}, *}$ Elinor C. Spencer, ${ }^{\mathrm{a}}$ Lynn A. Boatner ${ }^{\mathrm{b}}$ \\ ${ }^{a}$ Dept. of Geosciences, Virginia Tech, Blacksburg, VA 24061, U.S.A. \\ ${ }^{\mathrm{b}}$ Materials Science and Technology Division, Oak Ridge National Laboratory, Oak Ridge, Tennessee 37831, U.S.A.
}

\begin{abstract}
Accurate elastic constants for gadolinium phosphate $\left(\mathrm{GdPO}_{4}\right)$ have been measured by singlecrystal high-pressure diffraction methods. The bulk modulus of $\mathrm{GdPO}_{4}$ determined under hydrostatic conditions, $128.1(8) \mathrm{GPa}\left(\mathrm{K}^{\prime}=5.8(2)\right)$, is markedly different from that obtained with $\mathrm{GdPO}_{4}$ under non-hydrostatic conditions (160(2) GPa), which indicates the importance of shear stresses on the elastic response of this phosphate. High pressure Raman and diffraction analysis indicate that the $\mathrm{PO}_{4}$ tetrahedra behave as rigid units in response to pressure and that contraction of the $\mathrm{GdPO}_{4}$ structure is facilitated by bending/twisting of the Gd-O-P links that result in increased distortion in the $\mathrm{GdO}_{9}$ polyhedra.
\end{abstract}

\section{Introduction}

Rare-earth minerals with the general formula, $\mathrm{MPO}_{4}(\mathrm{M}=$ trivalent lanthanide cation) are widespread accessory minerals that are frequently doped with uranium and/or thorium. Phosphates of this class that incorporate the larger rare-earth cations $(\mathrm{M}=\mathrm{Ce}, \mathrm{Pr}, \mathrm{Nd}, \mathrm{Pm}, \mathrm{Sm}$, $\mathrm{Eu}$, and $\mathrm{Gd}$ ) adopt a monoclinic structure and are isostructural with the cerium phosphate mineral monazite, whereas phosphates with smaller cations $(\mathrm{M}=\mathrm{Tb}$, Dy, Ho, Er, Tm, $\mathrm{Yb}$ and $\mathrm{Lu}$ ) are isostructural with xenotime (tetragonal, $I 4_{1} / a m d$ ). The doping of these phosphates with radioactive elements, which have half-lives on the order of billions of years, has led to their use as geochronometers for the dating of geological formations. ${ }^{1}$ There has also been a developing interest in utilising simple phosphates as possible solid state repositories for nuclear waste, ${ }^{2}$ and due to its high neutron absorption capabilities, $\mathrm{GdPO}_{4}$ has been proposed as an inclusion compound for the construction of spent nuclear fuel canisters. ${ }^{3}$ The development of simple phosphate compounds for addressing this important socio-political problem has been driven by their excellent resistance to temperature and pressure fluctuations and low solubility and ion leaching. Although there has been considerable focus on determining the elastic properties of $\mathrm{MPO}_{4}$ compounds, ${ }^{4}$ the actual structural response of these materials to pressure remains underexplored. This important oversight must be addressed if these materials are to find practical application as safe and effective repository materials. In this contribution, we report the elastic constants of $\mathrm{GdPO}_{4}$ and correlate these with the underlying changes in the crystal structure in response to hydrostatic pressure. Structural studies of this kind will lead to a greater understanding of the origins of the stability of $\mathrm{MPO}_{4}$ compounds and facilitate progress in their development for nuclear waste storage applications.

$\mathrm{GdPO}_{4}$ crystallises in the monoclinic space group $P 2_{1} / n$. The asymmetric unit comprises a single $\mathrm{Gd}^{3+}$ ion and one $\mathrm{PO}_{4}{ }^{3-}$ anion. The phosphate anion has the expected tetrahedral coordination with no significant distortion. The coordination geometry of the nine-coordinate 
$\mathrm{Gd}^{3+}$ ion is best described as a severely distorted mono-capped square antiprism (MCSAP). The packing arrangement of the polyhedra is complex (Fig. 1a) but can be described as chains of alternating $\mathrm{PO}_{4}$ and $\mathrm{GdO}_{9}$ polyhedra that, in the standard $P 2_{1} / n$ setting, lie parallel to the [100] direction. These chains are then cross-linked via bridging oxygen atoms between the $\mathrm{Gd}^{3+}$ ions. This packing arrangement results in each $\mathrm{Gd}^{3+}$ being coordinated to seven $\mathrm{PO}_{4}$ tetrahedra, five of which are vertex sharing, while the remaining two are edge sharing. Furthermore, each $\mathrm{Gd}^{3+}$ ion shares common edges with six neighbouring $\mathrm{Gd}^{3+}$ cations (Fig. 1b).

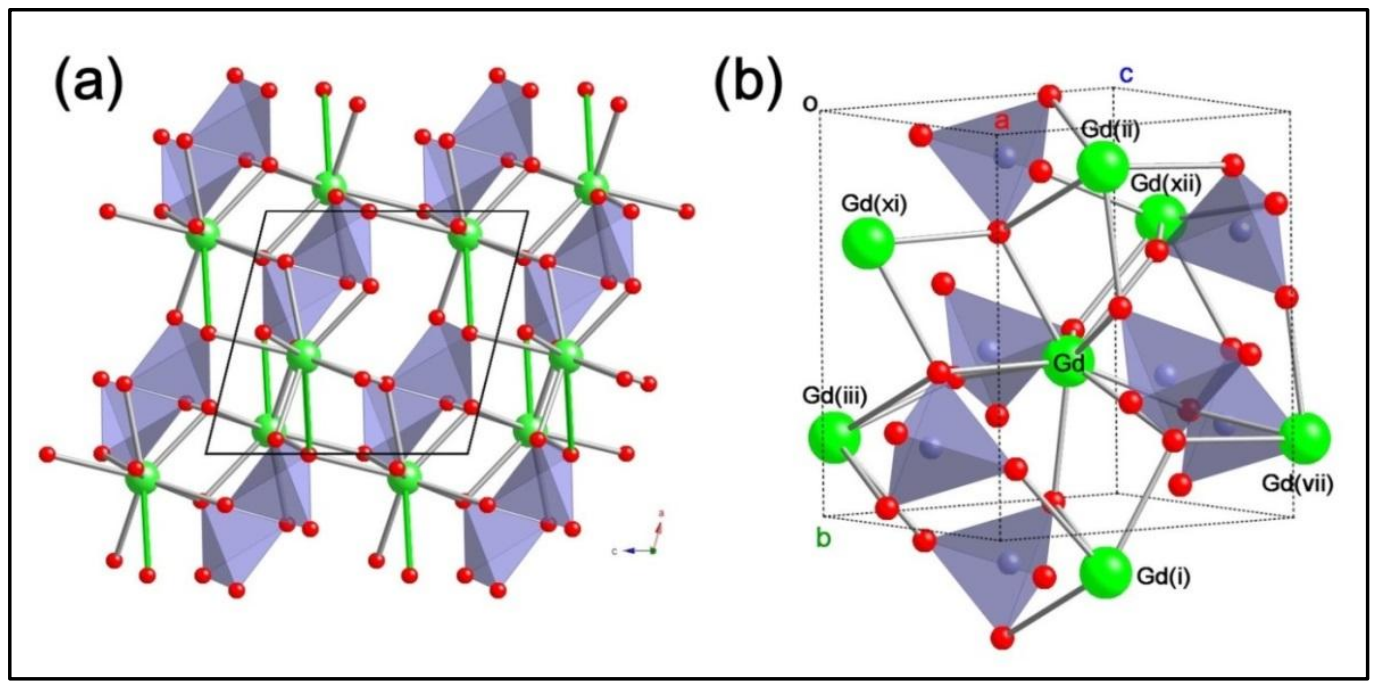

Figure 1. (a) The crystal structure of $\mathrm{GdPO}_{4}$ as viewed along the [010] direction; (b) the secondary coordination sphere of the $\mathrm{Gd}^{3+}$ ion in $\mathrm{GdPO}_{4}$. Each ion is coordinated to seven $\mathrm{PO}_{4}$ tetrahedra and has six edge-sharing interactions with neighboring $\mathrm{Gd}^{3+}$ ions. Green: $\mathrm{Gd}$; red: O; light grey: P. Green struts represent the bond between $\mathrm{Gd}^{3+}$ ions and the capping oxygen atoms of the MCSAP, all other covalent bonds are shown in grey. Symmetry codes: (i) $-\mathrm{x}+1 / 2, \mathrm{y}+1 / 2,-\mathrm{z}+3 / 2$; (ii) $-\mathrm{x}+1 / 2, \mathrm{y}-1 / 2,-\mathrm{z}+3 / 2$; (iii) $\mathrm{x}-1 / 2,-\mathrm{y}+3 / 2, \mathrm{z}-1 / 2$; (vii) $\mathrm{x}+1 / 2,-\mathrm{y}+3 / 2, \mathrm{z}+1 / 2$; (xi) -x,1y,1-z; (xii) -x, 1-y, 2-z.

\section{Experimental Methods}

Synthesis: Single crystals of $\mathrm{GdPO}_{4}$ were grown by a high-temperature solution (flux) growth method that has been described in detail previously. ${ }^{5}$ For the growth of single crystals of $\mathrm{GdPO}_{4}$, high-purity $\mathrm{Gd}_{2} \mathrm{O}_{3}$ was combined with lead hydrogen phosphate in a $50 \mathrm{ml}$ platinum crucible that was then heated to $1360{ }^{\circ} \mathrm{C}$ for several days. At $1360{ }^{\circ} \mathrm{C}$, lead pyrophosphate, $\mathrm{Pb}_{2} \mathrm{P}_{2} \mathrm{O}_{7}$, forms via the decomposition of the lead hydrogen phosphate that then reacts with $\mathrm{Gd}_{2} \mathrm{O}_{3}$ to form $\mathrm{GdPO}_{4}$ dissolved in a solution of molten $\mathrm{Pb}_{2} \mathrm{P}_{2} \mathrm{O}_{7}$. Single crystals of $\mathrm{GdPO}_{4}$ grow from the flux as the system is cooled to $\sim 900{ }^{\circ} \mathrm{C}$. At this temperature, the growth furnace is rapidly cooled to room temperature, and the crystals are removed from the solidified flux by boiling the Pt crucible and growth charge in nitric acid for several weeks. The entire growth process requires a total period of over two months. 
High-pressure diffraction $(H P-X R D)$ : A single crystal of $\mathrm{GdPO}_{4}\left(81 \times 153 \times 248 \mu \mathrm{m}^{3}\right)$ was loaded into a sample chamber approx. $290 \times 100 \mu \mathrm{m}^{2}$ in size that was drilled into a steel gasket that was fitted into a standard ETH diamond anvil cell (DAC). ${ }^{6}$ A quartz crystal was loaded into the cell with the sample crystal to act as a pressure calibrant. ${ }^{7}$ Diffraction data were first collected under ambient conditions before a 4:1 MeOH:EtOH mixture was added to the DAC. This mixture acts as a pressure-transmitting medium and is known to remain hydrostatic up to 9.8 $\mathrm{GPa}^{8}$

Precise unit cell parameters for both the sample and quartz crystal were obtained from data collected on a Huber diffractometer equipped with a Eulerian cradle and point detector. The method is based on the 8-position centering of 10-25 intense diffraction maxima within the $9^{\circ}<2 \theta<33^{\circ}$ range. ${ }^{9}$ Data for structural analysis were collected at select pressures with an XCalibur-1 (Rigaku Oxford Diffraction) diffractometer equipped with a CCD detector and operating with monochromatic Mo-K $\mathrm{K}_{\alpha}$ radiation $(\lambda=0.71073 \AA)$. The data were processed with standard instrument software. Sample and DAC absorption effects were corrected for with ABSORB-7. ${ }^{10}$ Diamond dips and statistical corrections were performed with AVERAGE (R. J. Angel, 2003-2011). High-pressure structural models for $\mathrm{GdPO}_{4}$ were determined from the refinement, against the pressure data, of a structural model determined with the crystal in air and under ambient conditions (see SI for details). This was possible because the sample underwent no phase transitions over the pressure range investigated. Refinement was against $F^{2}$ by fullmatrix least-squares refinement techniques. Due to restricted access to reciprocal space due to spatial restrictions imposed by the DAC all atoms in the high-pressure structural models were refined isotropically. Key data and refinement parameter for the HP-XRD analysis are listed in Table 1.

Table 1. Data and refinement parameters for $\mathrm{GdPO}_{4}$ as a function of pressure.

\begin{tabular}{|c|c|c|c|c|}
\hline & $\sim 1$ bar & $2.903(4) \mathrm{GPa}$ & 5.372(6) GPa & 7.062(6) GPa \\
\hline Crystal system & monoclinic & monoclinic & monoclinic & monoclinic \\
\hline Space Group & $P 2_{1} / n$ & $P 2_{1} / n$ & $P 2_{1} / n$ & $P 2_{1} / n$ \\
\hline$a(\AA)$ & $6.33571(12)$ & $6.29790(12)$ & $6.26830(11)$ & $6.24937(12)$ \\
\hline $\boldsymbol{b}(\AA)$ & $6.84840(17)$ & $6.80019(19)$ & $6.7632(2)$ & $6.7397(2)$ \\
\hline$c(\AA)$ & $6.6516(3)$ & $6.5923(2)$ & $6.5466(2)$ & $6.5189(2)$ \\
\hline$\beta\left({ }^{0}\right)$ & $104.023(2)$ & $103.833(2)$ & $103.672(2)$ & $103.563(2)$ \\
\hline$V\left(\AA^{3}\right)$ & $280.008(14)$ & $274.139(13)$ & $269.674(13)$ & 266.912(14) \\
\hline$\rho\left(\mathrm{g} / \mathrm{cm}^{3}\right)$ & 5.983 & 6.111 & 6.212 & 6.277 \\
\hline Collected reflns & 1022 & 1948 & 1915 & 1821 \\
\hline $\mathbf{R}_{\text {int }}$ & 0.059 & 0.070 & 0.071 & 0.067 \\
\hline Data/parameters & $357 / 26$ & $460 / 26$ & $449 / 26$ & $438 / 26$ \\
\hline$S$ & 1.073 & 1.026 & 1.030 & 0.999 \\
\hline R1 $(I>2 \sigma I)$ & 0.0451 & 0.0433 & 0.0442 & 0.0418 \\
\hline$w \mathbf{R} 2(\mathrm{I}>2 \sigma \mathrm{I})$ & 0.1016 & 0.0958 & 0.1079 & 0.0945 \\
\hline
\end{tabular}


High-pressure Raman spectroscopy: A second $\mathrm{GdPO}_{4}$ crystal was loaded into a Brillouin-Raman (BR-series) DAC (High-Pressure Diamond Optics, Inc., U.S.A) fitted with two type-IIa diamonds, both $600 \mu \mathrm{m}$ in diameter. These diamonds, along with others in their class, have very low levels of impurities and therefore display negligible photoluminescence. The sample chamber $(\sim 300 \mu \mathrm{m}$ in diameter) was drilled in a steel gasket that was fitted between the diamonds of the DAC. A ruby ball was also loaded into the DAC for pressure calibration measurements. ${ }^{11}$ A 4:1 MeOH:EtOH mixture was added to the chamber to act as the pressure medium. High-pressure Raman spectra were recorded on a JY Horiba LabRam HR spectrometer fitted with a $40 \mathrm{x}$ objective and equipped with a red laser $(\lambda=632.817 \mathrm{~nm})$. The spectral slits were $150 \mu \mathrm{m}$ in size, and the confocal hole was set to $400 \mu \mathrm{m}$. The spectrograph grating had 1200 grooves $/ \mathrm{mm}$. Each data collection comprised two scans, each $40 \mathrm{~s}$ in duration. The photoluminescence signals (at ca. $694 \mathrm{~nm}$ ) of the ruby were measured under the same experimental conditions as the sample data collections - except that the scans lengths were varied to adjust the signal intensity when required.

\section{Results \& Discussion}

\section{Elastic Properties}

Figure 2 shows the pressure dependence of the unit cell volume of $\mathrm{GdPO}_{4}$, as well as the normalized pressure $\left(F_{e}\right)$ versus the Eulerian strain $\left(f_{e}\right)$ curves, and Figure 3 shows the changes in the unit cell parameters of $\mathrm{GdPO}_{4}$ as a function of pressure. The $F_{\mathrm{e}} v s$. $f_{\mathrm{e}}$ plot emphasizes small structural changes that may occur in response to pressure. All these plots show that $\mathrm{GdPO}_{4}$ undergoes no phase transitions over the investigated pressure range $(0-7 \mathrm{GPa})$ and that the compression mechanisms operating in this material are fully reversible.

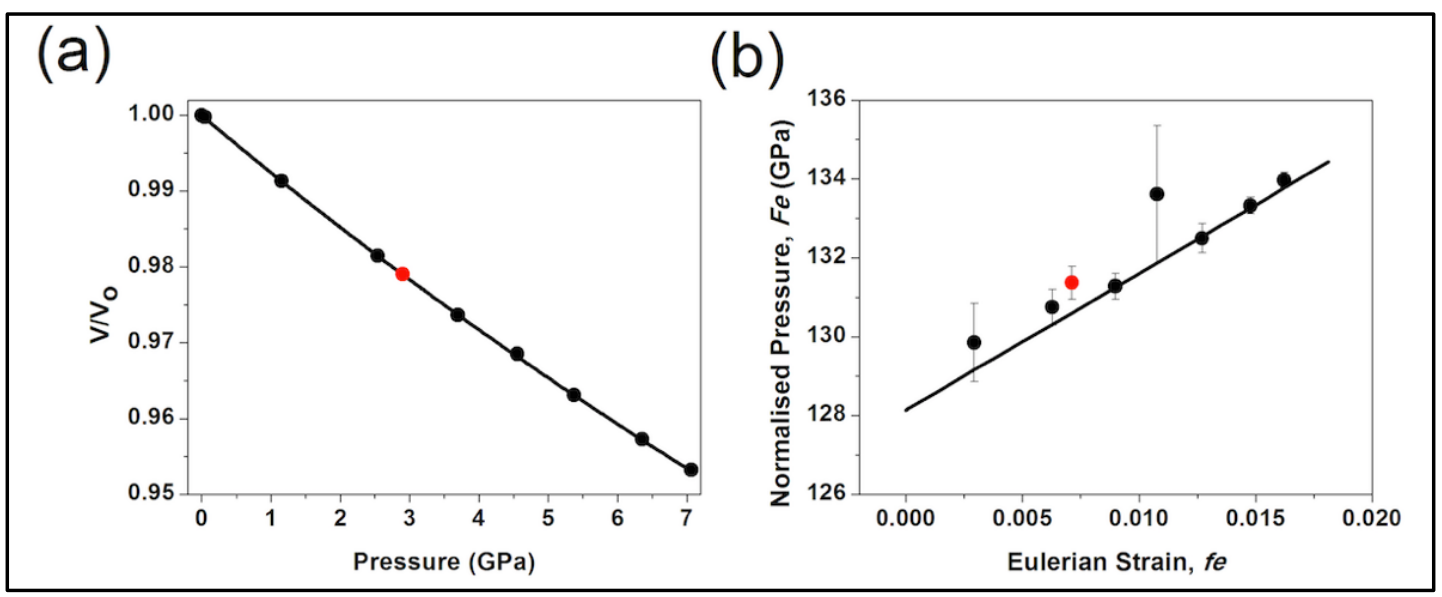

Figure 2. (a) Volume ratio vs. pressure for $\mathrm{GdPO}_{4}$. Error bars are smaller than the data points; (b) $\mathrm{F}_{\mathrm{e}}$ vs. $\mathrm{f}_{\mathrm{e}}$ plot. The black curves represent the $3^{\text {rd }} \mathrm{BM}-\mathrm{EoS}$ fits to the data. Data recorded during both pressure increase and decrease are shown as solid black and red circles, respectively. 


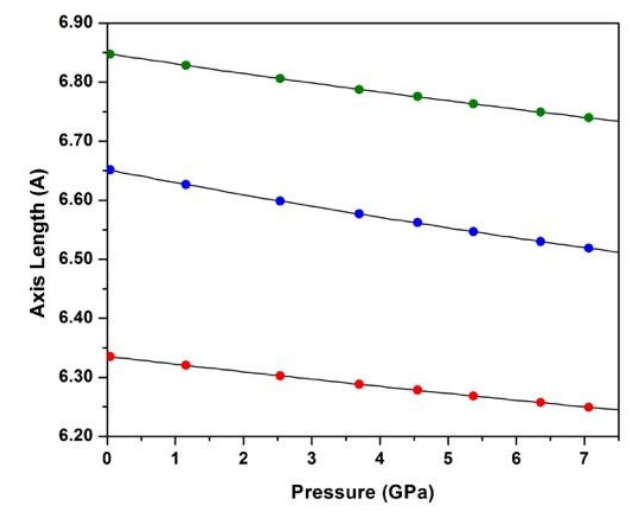

Figure 3. Unit cell parameters vs. pressure for $\mathrm{GdPO}_{4}$. Red: $a$-axis; green: $b$-axis; blue: $c$-axis. The black curves represent the $3^{\text {rd }}$ BM-EoS fits to the data. Error bars are smaller than the symbols.

Each of these curves was fit with a $3^{\text {rd }}$-order Birch-Murnaghan equation of state $(\mathrm{BM}-\mathrm{EoS})^{12}$ from which the elastic constants were derived (Table 2). $\mathrm{GdPO}_{4}$ is reasonably incompressible with a unit cell volume decrease of only $4.68 \%$ over the 0-7.062(6) GPa pressure range, and has a bulk modulus $\left(K_{0}\right)$ of $128.1(8) \mathrm{GPa}\left(\mathrm{K}^{\prime}=5.8(2)\right)$. A previously documented high-pressure powder diffraction study on synthetic $\mathrm{GdPO}_{4}$, conducted up to $30 \mathrm{GPa}$, reported a significantly higher bulk modulus of $160(2) \mathrm{GPa} .{ }^{13}$ However, these authors used neon as their pressure transmitting medium, which solidifies at $\sim 5 \mathrm{GPa}$. Above this pressure their $\mathrm{GdPO}_{4}$ sample is likely to have experienced deviatoric stresses that are known to significantly influence the highpressure behavior of rare-earth orthophosphates and other crystalline materials. ${ }^{14}$ It is therefore not unreasonable that our bulk modulus value, which was determined from data collected under hydrostatic conditions, varies significantly from theirs. This result highlights the critical need to understand the sample stress state (applied and internal) when determining the elastic properties of $\mathrm{MPO}_{4}$ compounds of this class. Consistent with the high bulk modulus for $\mathrm{GdPO}_{4}$ the axial moduli $\left(M_{0}\right)$ are also large, however the values of $M_{0}$ show a trend of decreasing axial compression in the order: [001] > [010] > [100]. This suggests that the chains of alternating polyhedra that lie parallel to [100] are more rigid than the directions perpendicular to them. This is consistent with the cross-linking of the chains through edge-sharing $\mathrm{GdO}_{9}$ polyhedra resulting in flexibility in the [001] and [010] directions.

Table 2. Elastic constants for $\mathrm{GdPO}_{4}$ derived from $3^{\text {rd }}$-order $\mathrm{BM}-\mathrm{EoS}$ fits to the variable pressure unit cell parameters.

\begin{tabular}{|c|c|c|c|c|c|}
\hline & & & $\boldsymbol{a}$-axis & $\boldsymbol{b}$-axis & $\boldsymbol{c}$-axis \\
\hline $\boldsymbol{V}_{\mathbf{0}}\left(\mathbf{\AA}^{\mathbf{3}}\right)$ & $280.017(18)$ & $\boldsymbol{X}_{\mathbf{0}}(\mathbf{\AA})$ & $6.33584(11)$ & $6.84839(18)$ & $6.6519(2)$ \\
\hline $\boldsymbol{K}_{\mathbf{0}}(\mathbf{G P a})$ & $128.1(8)$ & $\boldsymbol{M}_{\mathbf{0}}(\mathbf{G P a})$ & $463(3)$ & $389(3)$ & $297(2)$ \\
\hline $\boldsymbol{K}^{\prime}$ & $5.8(2)$ & $\boldsymbol{M}^{\prime}$ & $15.2(9)$ & $15.8(11)$ & $16.2(8)$ \\
\hline $\boldsymbol{K}^{\prime \prime}\left(\mathbf{G P a}^{-\mathbf{1}}\right)$ & -0.06991 & $\boldsymbol{M}^{\prime \prime}\left(\mathbf{G P a}^{-\mathbf{1}}\right)$ & -0.11929 & -0.15689 & -0.21983 \\
\hline
\end{tabular}




\section{Compression Mechanism:}

Due to the relatively high bulk modulus of $\mathrm{GdPO}_{4}$ only small structural changes occur over the pressure range investigated here. Indeed, the subtlety of the pressure-induced changes can be seen visually in Figure 4. This figure shows an overlay of the gadolinium coordination sphere at room-pressure and at 7.062(6) GPa, and it is immediately clear that there are no significant alterations to the structure between these two pressure points. Therefore, to analyze how the structure changes, it is necessary to consider the effect of pressure on the larger features of the $\mathrm{GdPO}_{4}$ structure rather than the small changes to individual angles/bonds, the magnitudes of which are within the estimated standard deviation for these parameters. To achieve this objective, we will consider the pressure-induced alterations to three components of the structure in turn: (i) the phosphate tetrahedra, (ii) the gadolinium polyhedra, and (iii) the inter-polyhedral distances.

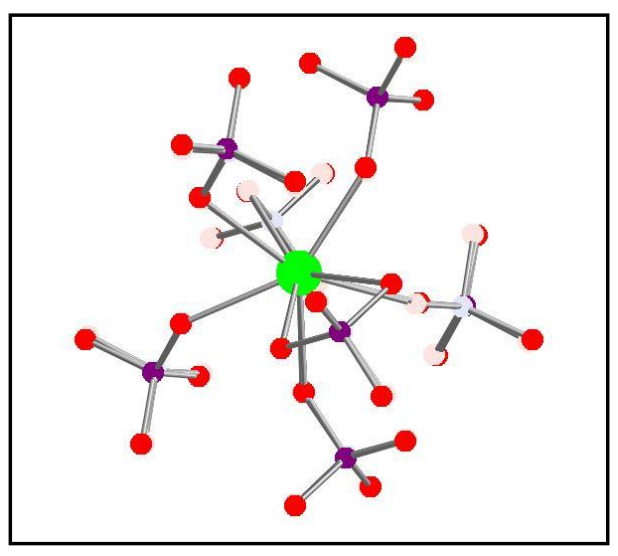

Figure 4. An overlay of the Gd atom's coordination sphere at $~ 1$ bar and 7.062(6) GPa. The $\sim 1$ bar structure is shown in dark purple and red, while the 7.062(6) GPa structure is shown in light pink and blue.

To discern whether there are any changes to the phosphate tetrahedra in response to pressure, it is useful to first calculate distortion parameters for these polyhedra. The distortion parameters of interest are the elongation parameter $\left(\left\langle\lambda_{\text {tet }}\right\rangle\right)$ that quantifies the distortion to the tetrahedra due to variation in the bond lengths, and the bond angle variance $\left(\sigma_{(\mathrm{tet})}{ }^{2}\right)$ that quantifies the distortion due to variation in the bond angles within the tetrahedra. ${ }^{15}$ For an ideal tetrahedron $\left(\left\langle\lambda_{\text {tet }}\right\rangle\right)=1$ and $\left(\sigma_{(\text {tet })}{ }^{2}\right)=0$. Table 3 reports the values of these distortion parameters at various pressures. The $\mathrm{PO}_{4}$ tetrahedra contain a fair amount of distortion. This is primarily due to deviations in the bond angles, as evident from the variance parameter that ranges from 22(3) to 29(4); while the elongation parameter remains constant at 1.01, and implies that the bond lengths do not vary greatly within the tetrahedra. However, there is no statistically relevant change in either distortion parameter with increasing pressure. Therefore, the phosphate tetrahedra can be treated as rigid units when analyzing the overall compression mechanisms of $\mathrm{GdPO}_{4}$ up to 7.062(6) GPa. 
Table 3. Distortion parameters for the phosphate tetrahedra at select pressures.

\begin{tabular}{|c|c|c|c|c|}
\hline Pressure $(\mathbf{G P a})$ & $\boldsymbol{V}_{\text {tet }}\left(\AA^{\mathbf{3}}\right)$ & $\boldsymbol{l}_{\mathbf{0}}(\stackrel{\AA}{\mathbf{A}})$ & $\left\langle\lambda_{\text {tet }}\right\rangle$ & $\left(\boldsymbol{\sigma}_{\text {tet }}\right)^{\mathbf{2}}$ \\
\hline$\sim 1$ bar & 1.826 & 1.527 & 1.01 & $29(4)$ \\
\hline $2.903(4)$ & 1.816 & 1.524 & 1.01 & $23(3)$ \\
\hline $5.372(6)$ & 1.807 & 1.521 & 1.01 & $29(3)$ \\
\hline $7.062(6)$ & 1.820 & 1.525 & 1.01 & $26(3)$ \\
\hline
\end{tabular}

The gadolinium polyhedra were also analyzed for pressure-induced structural changes. However, before any distortion parameters could be calculated the geometry of the polyhedra had to be determined. The central gadolinium ion is coordinated to nine oxygen atoms with bond lengths ranging from 2.326(10)-2.788(13) $\AA$; eight of these nine bonds are $2.45 \AA$ while one bond is $\sim 2.75 \AA$. The $\mathrm{GdO}_{9}$ coordination geometry is similar to that of a heavily distorted monocapped square antiprism (MCSAP, Fig. 5a). To date, no distortion parameters have been defined for a MCSAP polyhedron, so we will use the following parameters to quantify the distortion of the single symmetry independent $\mathrm{GdO}_{9} \mathrm{MCSAP}$ in $\mathrm{GdPO}_{4}$ : (i) plane centroid $\cdots$ centroid distance,

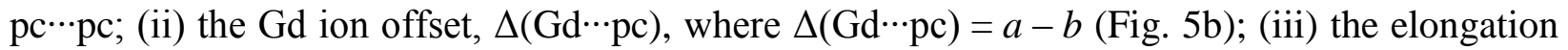
parameter, $\left\langle\lambda_{\text {sap }}\right\rangle$, which quantifies the distortion of the square antiprism due to variations in the $\mathrm{M}-\mathrm{O}$ bond lengths (Eq. 1). This is equivalent to $\left\langle\lambda_{\text {tet }}\right\rangle$ (vide supra); and (iv) the angular variance, $\sigma_{(\text {mcsap })}{ }^{2}$, which is equivalent to $\sigma_{(\text {(tet) }}{ }^{2}$, and is calculated with Equation 2:

$$
\begin{gathered}
<\lambda_{\text {sap }}>=\frac{1}{8} \sum_{i=1}^{4}\left(\frac{{ }^{t} l_{i}}{{ }^{t} l_{0}}\right)^{2}+\frac{1}{8} \sum_{i=1}^{4}\left(\frac{{ }^{b} l_{i}}{{ }^{b} l_{0}}\right)^{2} \\
\sigma_{(\text {mcsap })}{ }^{2}=\frac{1}{11} \sum_{i=4}^{4}\left({ }^{t} \theta_{i}-90\right)^{2}+\frac{1}{11} \sum_{i=4}^{4}\left({ }^{b} \theta_{i}-90\right)^{2}+\frac{1}{11} \sum_{i=4}^{4}\left({ }^{c} \theta_{i}-90\right)^{2}
\end{gathered}
$$

Equation 1.

Equation 2.

where ${ }^{t} l_{i}$ and ${ }^{b} l_{i}$ are the measured lengths of the Gd-O bonds that define the sides of the top and bottom square pyramids, respectively. ${ }^{t} l_{0}$ and ${ }^{b} l_{0}$ are the ideal $\mathrm{Gd}-\mathrm{O}$ distances for a perfect square pyramid with a volume equal to that of the top and bottom pyramid, respectively. Parameters ${ }^{t} \theta_{i}$ and ${ }^{b} \theta_{i}$ are the for $\mathrm{O}-\mathrm{pc}-\mathrm{O}$ angles within the bases of the top and bottom square pyramids, respectively; ${ }^{\mathrm{c}} \theta_{i}$ are the four $\mathrm{O}-\mathrm{pc}-\mathrm{O}$ angles between the oxygen atoms comprising the base of the top pyramid and the capping oxygen atom. These distortion parameters are shown in Table 4. For an ideal MCSAP, these parameters are $\left\langle\lambda_{\text {sap }}\right\rangle=1$ and $\sigma_{\text {(mcsap) }}{ }^{2}=0$. Under ambient pressure these parameters indicate that the MCSAP environment of the $\mathrm{Gd}^{3+}$ ion is heavily distorted, and this is primarily due to considerable deviation of the $\mathrm{O}-\mathrm{pc}-\mathrm{O}$ angles from $90^{\circ}$ rather than because of variation in the $\mathrm{Gd}-\mathrm{O}$ bond lengths. 


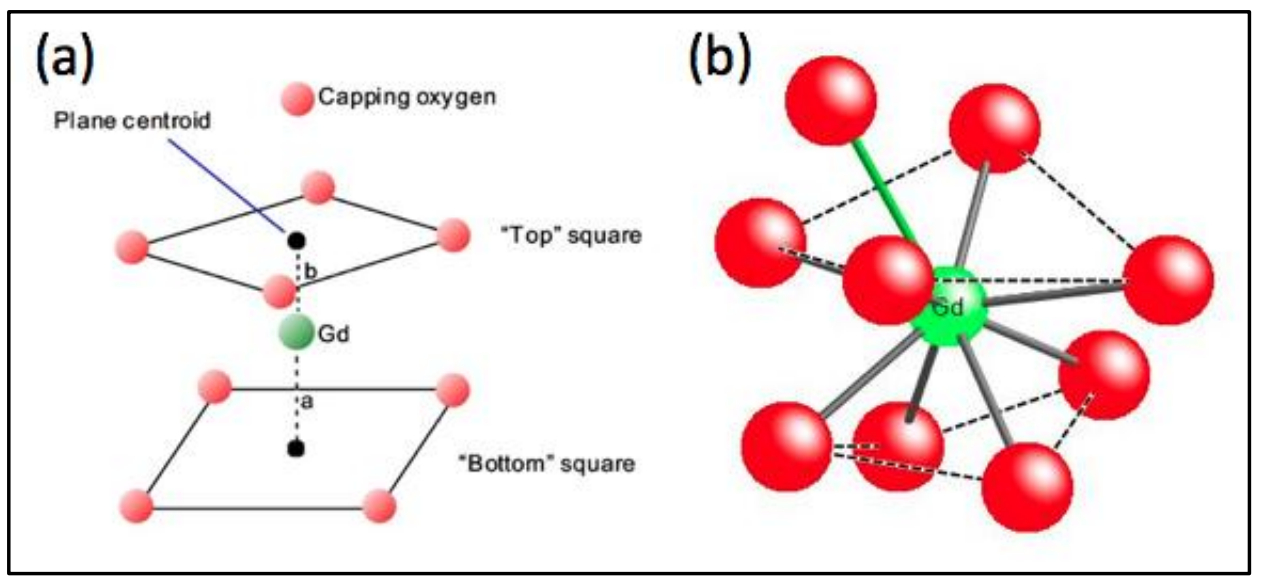

Figure 5. (a) Schematic representation of an ideal MCSAP; (b) Distorted MCSAP geometry of the $\mathrm{Gd}^{3+}$ ions in $\mathrm{GdPO}_{4}$ under ambient pressure. The $\mathrm{Gd}-\mathrm{O}$ bond involving the capping oxygen atom is shown in green.

Table 4. Distortion parameters for the gadolinium polyhedra at select pressures.

\begin{tabular}{|c|c|c|c|c|c|}
\hline Pressure (GPa) & 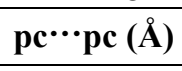 & $\Delta(\mathbf{G d} \cdots p c)(\AA)$ & ${ }^{t} l_{0} /{ }^{b} l_{0}(\AA)$ & $<\lambda_{\text {sap }}>$ & $\left(\sigma_{\text {mcsap }}\right)^{2}$ \\
\hline$\sim 1$ bar & $2.332(8)$ & $0.669(10)$ & $2.455 / 2.434$ & 1.00 & $631(6)$ \\
\hline $2.903(4)$ & $2.308(7)$ & $0.688(9)$ & $2.509 / 2.423$ & 0.97 & $644(5)$ \\
\hline $5.372(6)$ & $2.288(8)$ & $0.708(7)$ & $2.543 / 2.468$ & 0.93 & $631(5)$ \\
\hline $7.062(6)$ & $2.271(6)$ & $0.722(9)$ & $2.399 / 2.414$ & 1.00 & $637(5)$ \\
\hline
\end{tabular}

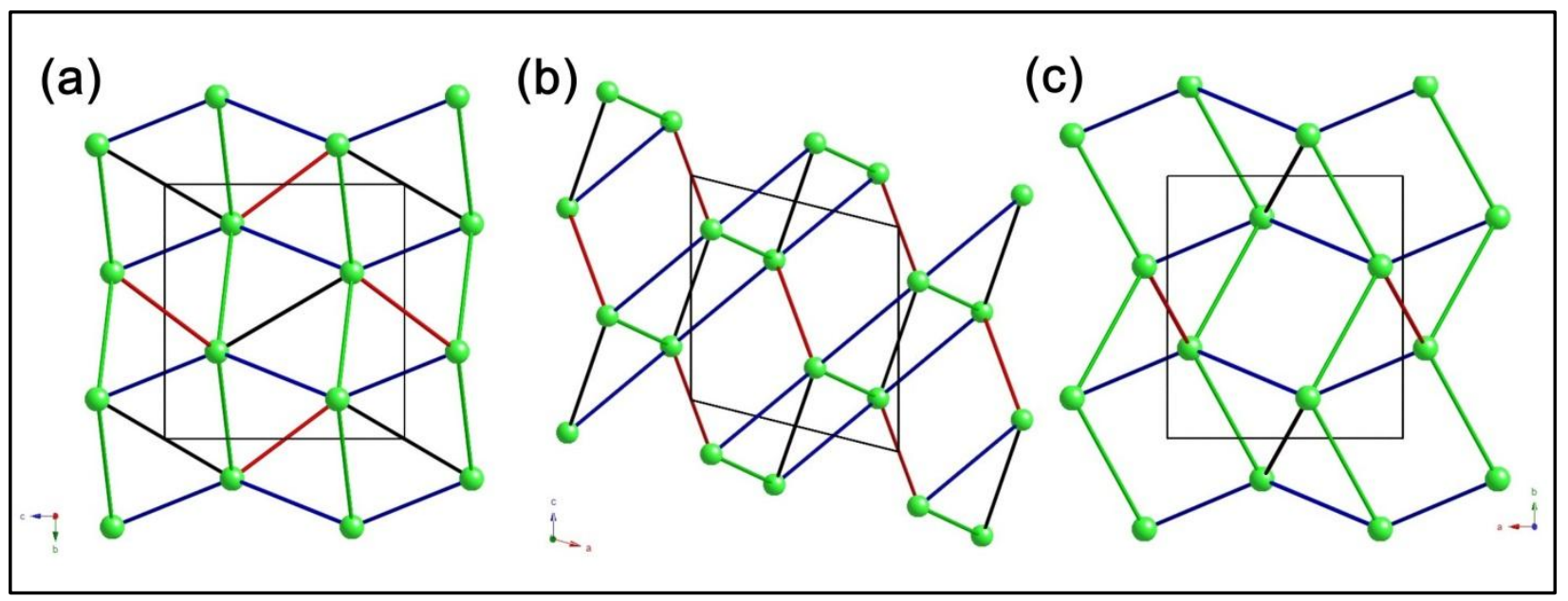

Figure 6. Pictorial representation of changes to the distances between neighbouring $\mathrm{Gd}^{3+}$ ions. Green struts represent a 0.104(1) $\AA$ decrease, black a 0.088(3) $\AA$ decrease, blue a 0.058(1) $\AA$ decrease, and the red struts represent no change in these distances between ambient and 7.062(6) GPa: (a) view along [100]; (b) view along [010]; (c) view along [001]. The unit cell is shown in grey. 
There are no statistically relevant changes to the parameters $\left\langle\lambda_{\text {sap }}\right\rangle$ and $\sigma_{(\text {mcsap })}{ }^{2}$ with pressure. However, there are statistically relevant changes to both the $\mathrm{pc} \cdots \mathrm{pc}$ distance and the $\Delta(\mathrm{Gd} \cdots \mathrm{pc})$ parameter with increasing pressure. As the pressure increases, the distance between the centroids of the square pyramid bases of the MCSAP decrease. Yet, the increase in the $\Delta(\mathrm{Gd} \cdots \mathrm{pc})$ parameter with pressure indicates that this is an asymmetrical compression (i.e. $\Delta \mathrm{a} \neq \Delta \mathrm{b}$ ). In fact, $\Delta \mathrm{b}>\Delta \mathrm{a}$ indicating that the $\mathrm{Gd}^{3+}$ ion is displaced towards the top plane of the MCSAP with increasing pressure. As shown in Figure 1, the $\mathrm{Gd}-\mathrm{O}$ bond involving the capping oxygen atoms of the MCSAP polyhedra are orientated approximately parallel to the [100] direction. Therefore, these alterations to the MCSAP polyhedra can be envisaged as facilitating compression in this direction.

To evaluate the overall pressure-induced changes to the $\mathrm{GdPO}_{4}$ structure it is beneficial to assess the secondary coordination sphere of the $\mathrm{Gd}^{3+}$ ions by considering the $\mathrm{Gd} \cdots \mathrm{Gd}$ distances between neighboring ions as these can be determined reliably from our high-pressure XRD data (Table 5). Figure 6 is a pictorial representation of how these pressure-induced decreases in the $\mathrm{Gd}^{\cdots} \mathrm{Gd}$ distances relate to the overall compression of the structure. The largest decrease occurs between $\mathrm{Gd}^{\cdots}{ }^{\text {edge }} \mathrm{Gd}^{\mathrm{i}}$ and $\mathrm{Gd}^{\cdots}{ }^{\text {edge }} \mathrm{Gd}^{\mathrm{ii}}$ (see Fig. $1 \mathrm{~b}$ for atom labeling scheme) is $0.104(1) \AA$ and these interactions are aligned approximately parallel to the [100] and [010] directions. The two intermediate decreases of $0.058(1) \AA$ (between $\mathrm{Gd}^{\cdots}{ }^{\text {edge }} \mathrm{Gd}^{\mathrm{iii}}$ and $\mathrm{Gd}^{\cdots}{ }^{\text {edge }} \mathrm{Gd}^{\text {vii }}$ ) and $0.088(3) \AA$ (between $\mathrm{Gd}^{\cdots}{ }^{\text {edge }} \mathrm{Gd}^{\mathrm{xi}}$ ) are aligned closest to the [010] and [001] directions. Lastly, there is no statistical change in the $\mathrm{Gd}^{\cdots} \mathrm{Gd}$ distances that would contribute to a reduction in the $c$-axis $\left(\mathrm{Gd}^{\cdots \cdot{ }^{\text {edge }}} \mathrm{Gd}^{\mathrm{xii}}\right)$. This analysis indicates that the combination of these structural changes corresponds well with the measured elastic constants for $\mathrm{GdPO}_{4}($ Table 2) that shows a decrease in compression in the order: [001] > [010] > [100].

Table 5. Distances between Gd atom positions at select pressures.

\begin{tabular}{|c|c|c|c|c|c|}
\hline Distance & $\sim 1$ bar & 2.903(4)GPa & 5.372(6) GPa & 7.062(6) GPa & $\Delta\left(\mathbf{P}_{\min } / \mathbf{P}_{\max }\right)$ \\
\hline $\mathrm{Gd} 1{ }^{\cdots}{ }^{\mathrm{edge}} \mathrm{Gd}^{\mathrm{i}}$ & $4.0075(7)$ & $3.9614(6)$ & $3.9268(7)$ & $3.9033(6)$ & $0.104(1)$ \\
\hline $\mathrm{Gd} 1 \ldots^{\mathrm{edge}} \mathrm{Gd}^{\mathrm{ii}}$ & $4.0075(7)$ & $3.9614(6)$ & $3.9268(7)$ & $3.9033(6)$ & $0.104(1)$ \\
\hline $\mathrm{Gd} 1 \cdots^{\text {edge }} \mathrm{Gd}^{\mathrm{iii}}$ & $4.2036(4)$ & $4.1771(4)$ & $4.1569(4)$ & $4.1454(4)$ & $0.058(1)$ \\
\hline $\mathrm{Gd} 1 \cdots^{\text {edge }} \mathrm{Gd}^{\text {vii }}$ & $4.2036(4)$ & 4.1771(4) & $4.1569(4)$ & $4.1454(4)$ & $0.058(1)$ \\
\hline Gd1 $\cdots^{\text {edge }} \mathrm{Gd}^{\mathrm{xi}}$ & $4.2212(18)$ & $4.1793(14)$ & $4.1509(13)$ & $4.1328(13)$ & $0.088(3)$ \\
\hline $\mathrm{Gd} 1 \cdots^{\text {edge }} \mathrm{Gd}^{\mathrm{xii}}$ & $4.0277(14)$ & $4.0277(12)$ & $4.0251(14)$ & $4.0240(12)$ & $0.00(4)$ \\
\hline
\end{tabular}

Symmetry codes: (i) $-\mathrm{x}+1 / 2, \mathrm{y}+1 / 2,-\mathrm{z}+3 / 2$; (ii) $-\mathrm{x}+1 / 2, \mathrm{y}-1 / 2,-\mathrm{z}+3 / 2$; (iii) $\mathrm{x}-1 / 2,-\mathrm{y}+3 / 2, \mathrm{z}-1 / 2$; (vii) $\mathrm{x}+1 / 2,-\mathrm{y}+3 / 2, \mathrm{z}+1 / 2$; (xi) -x,1-y,1-z; (xii) -x, 1-y, 2-z.

\section{Raman Spectroscopy:}

The Raman spectrum of $\mathrm{GdPO}_{4}$ recorded in air under ambient conditions is shown in Figure 7. The peak assignments have been made based on polarized Raman data reported by Silva et al. ${ }^{16}$ Peaks below $450 \mathrm{~cm}^{-1}$ are associated with the lattice modes of the crystal structure and the lowfrequency stretching vibrations of the $\mathrm{Gd}-\mathrm{O}$ bonds $\left(300-350 \mathrm{~cm}^{-1}\right)$. The $v_{1}, v_{2}, v_{3}$ and $v_{4}$ peaks are affiliated with internal modes of the $\mathrm{PO}_{4}$ tetrahedra. According to group theory, several of these modes are multiply degenerate: $1 v_{1}, 2 v_{2}, 3 v_{3}$ and $3 v_{4}$. 


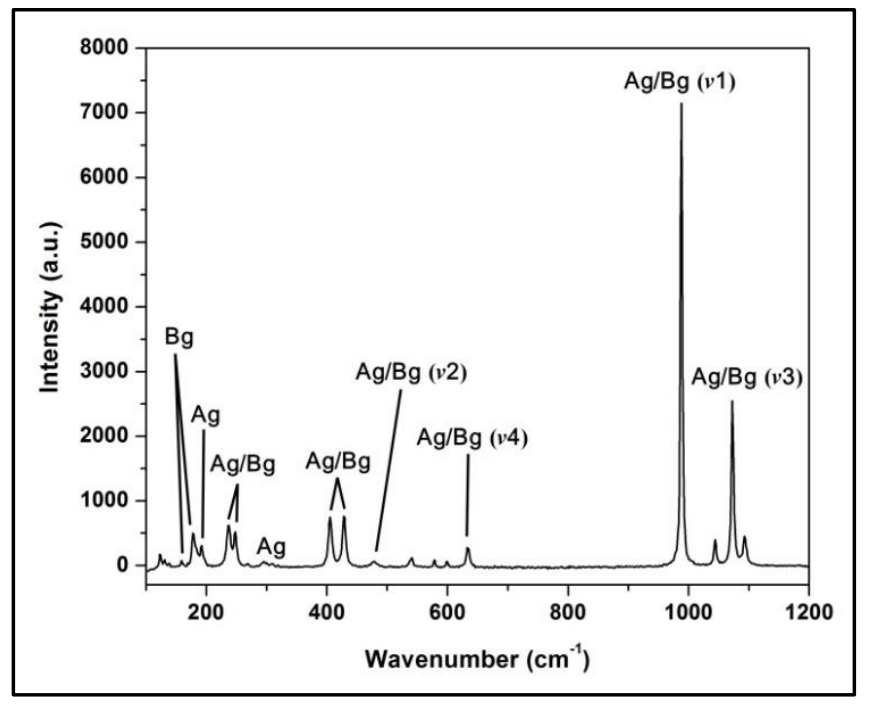

Figure 7. Raman spectrum of $\mathrm{GdPO}_{4}$ recorded in air under ambient conditions. Peak assignments are based on the polarized Raman results reported by Silva et al. ${ }^{16}$

Also present in the spectrum are unexpected peaks that are not predicted by theory. The origin of peaks that are in the regions of the $v_{3}$ and $v_{4}$ peaks has been a matter of contention. Several theories have been proposed for their occurrence including that they arise from splitting of the $v_{3}$ and $v_{4}$ fundamentals due to coupling between the $\mathrm{PO}_{4}$ tetrahedra - or that they are overtone modes. It has also been suggested that they arise due to the activation of the transverse and longitudinal optical components of the IR modes. ${ }^{16}$

The hypothesis that these extra peaks originate from the breaking of the degeneracy of the $v_{3}$ and $v_{4}$ mode has previously been discredited for two reasons: (i) the lack of evidence of any covalent character of the $\mathrm{Gd}-\mathrm{O}$ bond, which supports the idea that the $\left(\mathrm{PO}_{4}\right)^{3-}$ units should be considered as "free" (i.e. non-interacting) in the $\mathrm{GdPO}_{4}$ crystal structure; (ii) the fact that the doubly degenerate $v_{2}$ mode does not appear split, which would be expected if the other modes were split due to distortion of the $\mathrm{PO}_{4}$ tetrahedra. ${ }^{16,17}$ However, more recent DFT calculations performed on isostructural $\mathrm{CePO}_{4}$ and supported by experimental Raman studies have shown that these peaks are fundamental modes that arise from the local distortions in the $\mathrm{PO}_{4}$ tetrahedra that leads to the lifting of the degeneracies of the $\mathrm{P}-\mathrm{O}$ asymmetric stretching modes. ${ }^{18}$ This implies that the assumption that the $\left(\mathrm{PO}_{4}\right)^{3-}$ units can be regarded as "free" non-interacting units is flawed. Indeed, a systematic theoretical study of a broad range of $\mathrm{MXO}_{4}(\mathrm{M}=$ trivalent lanthanide ion; $\mathrm{X}=\mathrm{P}, \mathrm{As}$ ) compounds, including $\mathrm{GdPO}_{4}$, has unequivocally demonstrated that there is significant covalent character within the $\mathrm{M}-\mathrm{O}$ bonds. ${ }^{19}$ These results are entirely consistent with our crystallographic findings that demonstrate that the tetrahedra are strongly distorted in the $\mathrm{GdPO}_{4}$ structure (Table 3). This would not be expected if such units were truly structurally independent entities. With respect to the second objection, the $v_{2}$ peak is broad, certainly when compared to the sharp singly degenerate $v_{1}$ peak, and any fine splitting could easily be obscured. 


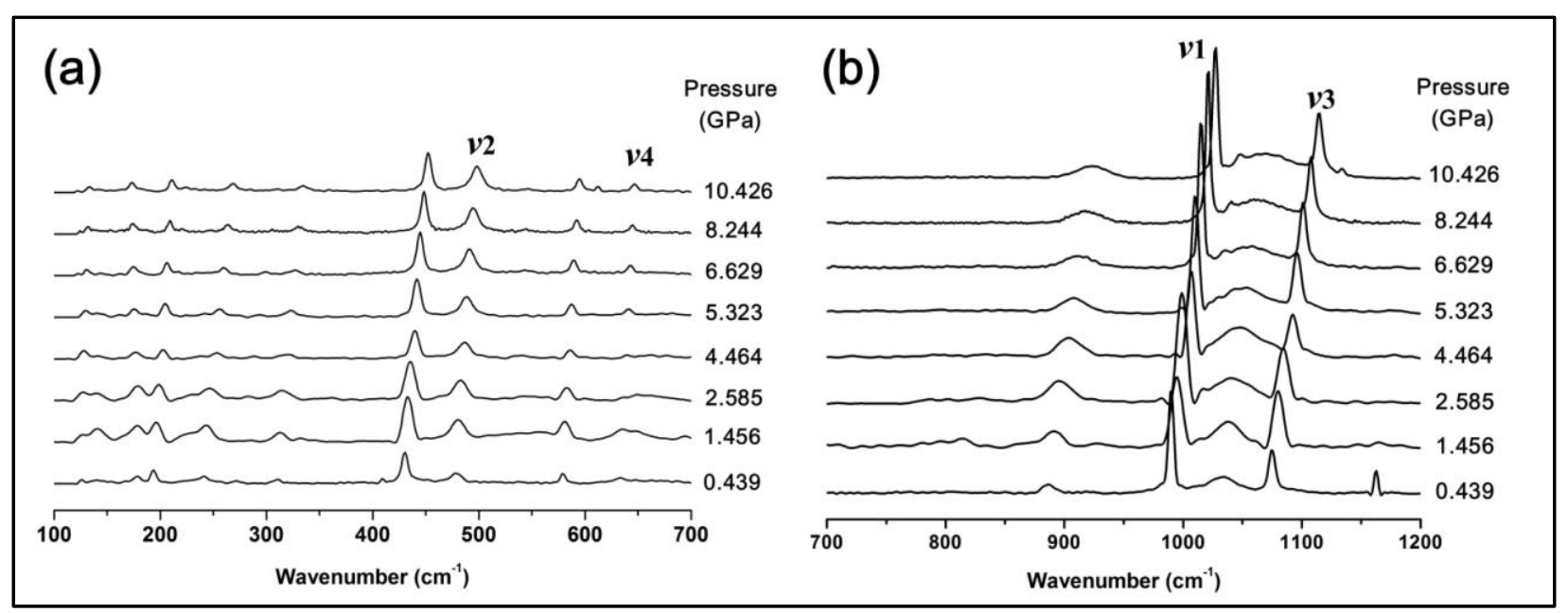

Figure 8. Variable-pressure Raman data for $\mathrm{GdPO}_{4}$ : (a) $100-700 \mathrm{~cm}^{-1}$ range; (b) $700-1200 \mathrm{~cm}^{-1}$ range. The peak at $\sim 1160 \mathrm{~cm}^{-1}$ in the spectrum recorded at $0.439 \mathrm{GPa}$ is an electronic spike and is not sample related. These spectra have been smoothed with a Savitzky-Golay function to enable spectral features to be seen clearly.

Variable-pressure Raman spectra of $\mathrm{GdPO}_{4}$ are presented in Figure 8. Consistent with our HP-XRD data (Figs. 2 and 3), these data show no evidence of any structural phase transitions over the pressure range investigated $(0.439-10.426 \mathrm{GPa})$. Within the hydrostatic regime $(P<9.8 \mathrm{GPa})$, the spectra are similar, however at $10.426 \mathrm{GPa}$ additional peaks appear adjacent to the $v_{3}$ peak $\left(\sim 1080 \mathrm{~cm}^{-1}\right)$ and between the $v_{2}$ and $v_{4}$ peaks $\left(580-640 \mathrm{~cm}^{-1}\right)$. This could suggest that the presence of shear stresses further distorts the $\mathrm{PO}_{4}$ units and potentially enhances the nondegeneracy of the internal $\mathrm{PO}_{4}$ stretching modes.

It is immediately apparent from Figure 8 that the $v_{3}$ asymmetric stretching mode of the $\mathrm{PO}_{4}$ tetrahedra is the only peak to show a statistically significant shift between 0.439 and $8.244 \mathrm{GPa}$ $\left(\Delta\left(v_{3}\right)=32.7(1) \mathrm{cm}^{-1}\right)$. This peak is not split in these spectra, presumably because of the reduction in data resolution relative to the spectrum collected with the sample in air (Fig. 7). It is also noticeable that the $v_{4}$ peak becomes more pronounced with increasing pressure. This indicates that the pressure-induced distortions that occur within $\mathrm{GdPO}_{4}$ impacts the asymmetric stretching of the $\mathrm{PO}_{4}$ tetrahedra. That said, our HP-XRD results (Table 2) and theoretical studies ${ }^{19}$ suggest that the $\mathrm{PO}_{4}$ units behave as rigid units under pressure, and therefore, we conclude that the changes in these modes are most likely a consequence of alterations in the $\mathrm{Gd}-\mathrm{O}-\mathrm{P}$ linkages (twisting or bending). One can envisage that this would result in the distortions to the $\mathrm{GdO}_{9}$ polyhedra that were evident in the HP-XRD data. It is rather unfortunate that the $\mathrm{Gd}-\mathrm{O}$ stretching modes that could potentially support or debunk this hypothesis are too poorly resolved in the Raman data to be analyzed.

\section{Conclusion}

In this contribution, we have presented a comprehensive high-pressure study of $\mathrm{GdPO}_{4}$. Our results have shown that the bulk modulus of $\mathrm{GdPO}_{4}$ measured under hydrostatic conditions $(128(8) \mathrm{GPa})$ is notably different from the previously reported value of 160(2) GPa measured 
under non-hydrostatic conditions. This finding suggests that $\mathrm{GdPO}_{4}$, and possibly other $\mathrm{MPO}_{4}$ materials are sensitive to shear stresses that could potentially influence not only their elastic properties but also their structural response to applied pressure. Moreover, accurate determination of the elastic constants for $\mathrm{GdPO}_{4}$ has shown that the axial compressions are anisotropic and increase in the order of: $[100]<[010]<[001]$.

Analysis of complementary high-pressure single-crystal diffraction and Raman data have established that the $\mathrm{PO}_{4}$ tetrahedra act as rigid entities under pressure, and compression is enabled through adjustments to the $\mathrm{O}-\mathrm{Gd}-\mathrm{P}$ linkages that result in subtle alterations to the $\mathrm{GdPO}_{9}$ polyhedra.

Importantly, this study has confirmed the resilience of $\mathrm{GdPO}_{4}$ to pressure, and has confirmed the suitability of $\mathrm{GdPO}_{4}$ as a candidate for inclusion in solid-state repository materials for highlevel nuclear waste storage.

\section{Acknowledgements}

N. L. R., K. M. H., and E. C. S. gratefully acknowledge support from the National Science Foundation (Grant No. EAR-1118691). K. M. H. and E. C. S. would also like to thank Drs. Carla Slebonick and Jing Zhou for their help and valuable insight on this project. Research at the Oak Ridge National Laboratory for one author (LAB) was supported by the U.S. Department of Energy, Office of Science, Basic Energy Sciences, Materials Sciences and Engineering Division.

\section{References}

[1] Grove, M.; Harrison, T.M.; Geology. 1999, 27, 487-490.

[2] Ewing, R.C.; Can. Min. 2001, 39, 697-715.

[3] P.A. Lessing.; A. W. Erickson.; J. Eur. Ceramic Soc. 2003, 23, 3049-3057.

[4] Clavier, N.; Podor, R.; Dacheux, N.; J. Eur. Ceramic Soc. 2011, 31, 941-976.

[5] Boatner, L.A. Phosphates: Geochemical, Geobiological, and Materials Importance; Hughes, J.M.; Kohn, M.; Rakovan, J., Eds., Mineralogical Society of America and the Geochemical Society: Washington, DC, 2002; Vol. 48, 87-122

[6] Miletich, R.; Allan, D.R.; Kuh, W.F. High-Temperature and High-Pressure Crystal Chemistry; Hazen, R.M., Downs, R.T., Eds.; Mineralogical Society of America and the Geochemical Society: Washington, DC, 2000; Vol. 41, pp. 445-519.

[7] Angel, R.J.; Allen, D.R.; Miletich, R.; Finger, L.W. J. Appl. Cryst. 1997, 30, 461-466.

[8] Angel, R.J.; Bujak, M.; Zhao, J.; Diego Gatta, G.; Jacobsen, S. D. J. Appl. Cryst. 2007, 40, 26-32.

[9] Angel, R.J.; Finger, L.W. J. Appl. Cryst. 2011, 44, 247-251.

[10] Angel, R.; Gonzalez-Platas, J. J. Appl. Cryst. 2013, 46, 252-254.

[11] Mao, H. K.; Bell, P. M.; Shaner, J.W.; Steinberg, D. J. J. Appl. Phys. 1978, 49, 3276-3283.

[12] Angel, R.J.; Gonzalez-Platas, J.; Alvaro, M. Z. Kristallogr. 2014, 229, 405-419.

[13] Lacomba-Perales, R.; Errandonea, D.; Meng, Y.; Bettinelli, M. Phys. Rev. B. 2010, 81, 064113. 
[14] Zhao, J.; Angel, R.J.; Ross, N.L. Appl. Cryst. 2010, 43, 743-751

[15] Gibbs, G.V.; Ribbe, P.H.; Robinson, K. Science 1971, 172, 567-570.

[16] Silva, E.N.; Ayala, A.P.; Guedes, I.; Paschoal, C.W.A.; Moreira, R.L.; Loong, C.K.; Boatner, L.A.; Op. Mat. 2006, 29, 224-230.

[17] Hezel, A.; Ross, S. D. Spectrochim. Acta, 1966, 22, 1949-1961.

[18] Ruschel, K.; Nasdala, L.; Kronz, A.; Hanchar, J. M.; Többens, D. M.; Škoda, R.; Finger, F.; Möller, A. Miner. Petrol. 2012, 105, 41-55.

[19] Li, H.; Zhang, S.; Zhou, S.; Cao, X. Inorg. Chem. 2009, 48, 4542-4548. 


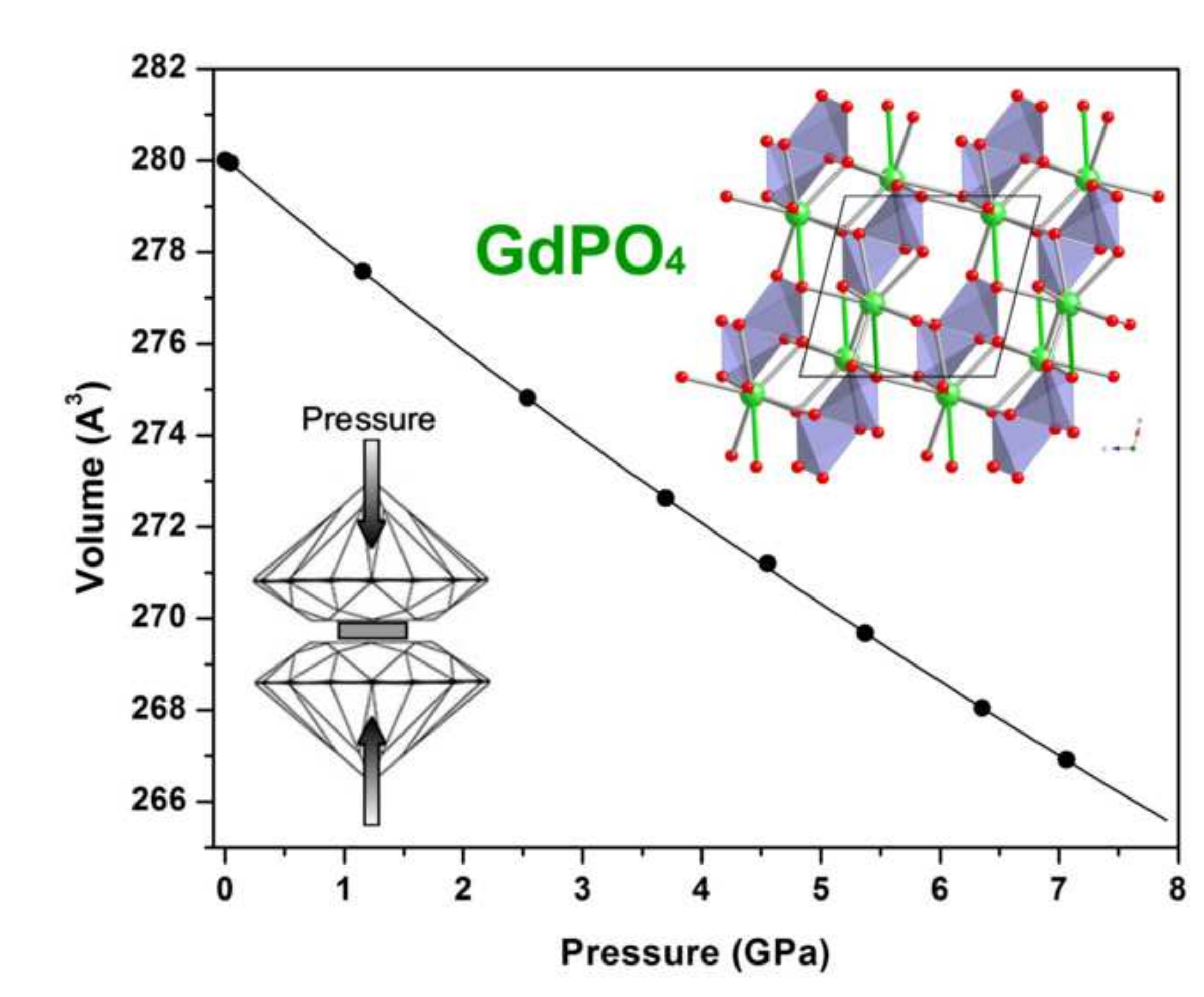

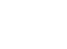

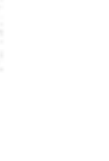




\section{Table of Contents Caption:}

A high-pressure single crystal diffraction study of $\mathrm{GdPO}_{4}$ with the monazite structure is presented. The elastic behaviour of rare-earth phosphates are believed to be sensitive to shear forces. The bulk modulus of $\mathrm{GdPO}_{4}$ measured under hydrostatic conditions is 128.1(8) GPa. Compression of the structure is facilitated by bending/twisting of the Gd-O-P links that result in increased distortion in the $\mathrm{GdO}_{9}$ polyhedra. 